\title{
La destitución de Fernando Lugo y Dilma Rousseff. Una comparación de ambas crisis democráticas desde la labor de las organizaciones regionales.*
}

\author{
por Alfonsina Gómez Martín **
}

\section{- Resumen}

En este artículo se describen dos casos de crisis democráticas en América Latina, el juicio político y destitución de los Presidentes Fernando Lugo en Paraguay (2012), y Dilma Rousseff en Brasil (2016). Con respecto a ambas crisis se comparan los procesos internos que las desencadenaron, y se apuntan qué mecanismos de protección democrática se implementaron por parte de las organizaciones regionales y qué factores influyeron en la implementación de dichos mecanismos. Aunque con matices particulares, en ambas crisis fueron factores internos los que provocaron el quiebre de las alianzas de gobierno y la consecuente conformación de mayorías legislativas con voluntad de destituir a los mandatarios. Mientras que en el caso paraguayo los mecanismos de protección democrática se aplicaron demostrando una convergencia en la acción del Mercado Común del Sur (MERCOSUR) y la Unión de Naciones Sudamericanas (UNASUR), pero no así de la Organización de Estados Americanos (OEA); en el caso de Brasil, donde las mismas organizaciones regionales eran competentes, los mecanismos no fueron activados salvo algunas acciones correspondientes a la fase de activación. Las asimetrías de poder en el marco de las organizaciones regionales y los distintos contextos regionales son factores que influyen de forma determinante en la aplicación o no de los mecanismos de protección democrática en cada caso particular.

\section{- Palabras Clave}

Crisis democráticas, organizaciones regionales, mecanismos de protección democrática, Paraguay, Brasil, juicio político

\section{@(1)}

Atribución 4.0 Internacional (CC BY 4.0)
Inglés | English

\section{The dismissal of Fernando Lugo and Dilma Rousseff. A comparison of both democratic crises from the work of regional organizations.}

\section{- Abstract}

This article describes two cases of democratic crisis in Latin America, the impeachments of Presidents Fernando Lugo in Paraguay (2012), and Dilma Rousseff in Brazil (2016). With respect to both crises, the internal processes that triggered them are compared, and it is pointed out that democratic protection mechanisms were implemented by regional organizations and what factors influenced the implementation of said mechanisms. Although with particular nuances, both crises were internal factors that led to the breakdown of government alliances and the consequent formation of legislative majorities with the intention of removing the presidents. While in the Paraguayan case the mechanisms of democratic protection were applied demonstrating a convergence in the action of the Common Market of the South (MERCOSUR) and the Union of South American Nations (UNASUR), but not the Organization of American States (OAS), in the case of Brazil, where the same regional organizations were competent, the mechanisms were not activated except for some actions corresponding to the activation phase. The asymmetries of power within the framework of regional organizations and the different regional contexts are factors that have a determining influence on the application or not of democratic protection mechanisms in each particular case.

\section{- Key words}

Democratic crisis, regional organizations, democratic protection mechanisms, Paraguay, Brazil, impeachment.

\footnotetext{
* El siguiente trabajo fue realizado retomando ideas plasmadas en la Tesis de Posgrado: "La acción de las organizaciones regionales en los casos de crisis democrática en Paraguay (2012) y Brasil (2016)”, realizada por la autora. Julio de 2017, Maestría en Integración y Cooperación Internacional, Centro de Estudios Interdisciplinarios, Universidad Nacional de Rosario.

${ }^{*}$ Licenciada en Relaciones Internacionales, Magister en Integración y Cooperación Internacional, Universidad Nacional de Rosario, alfonsina. gomez@hotmail.com.
} 


\section{Las crisis democráticas en la región, una historia de inestabilidad.}

Latinoamérica estuvo atravesada por diversas crisis democráticas a través de interrupciones abruptas de los mandatos constitucionales de sus presidentes a lo largo del siglo XX y lo que va del XXI. Resulta sorprendente la baja cantidad de mandatarios que pudieron terminar sus períodos de gobierno de acuerdo a lo establecido por las constituciones nacionales de los países que integran esta región (Valenzuela, 2004).

Ahora bien, se hace necesario distinguir los tipos de crisis democráticas ocurridas en la región. Por un lado, en la etapa previa al proceso de transición democrática sucedido durante la década de los años 80 y principios de los años 90, fueron los Golpes de Estado seguidos de largas dictaduras militares, los procesos recurrentes en los distintos países de América Latina. Sin embargo, luego de esta transición, las crisis democráticas y las interrupciones a los gobiernos electos se suceden a través de nuevas formas de crisis que se caracterizan por una baja participación de los militares, e involucran otras formas destituyentes que cuentan con el apoyo o aval de los mismos poderes que integran el sistema político democrático, como las presiones para generar renuncias anticipadas de presidentes, las crisis entre los poderes del Estado, los autogolpes y las destituciones a través de juicios políticos (Valenzuela, 2004; Pérez Liñán, 2009; Pignatta, 2011).

Estas nuevas situaciones de crisis que se presentan en la etapa posterior a la citada transición, han llamado la atención a la academia al punto de dedicárseles numerosas investigaciones. Es así como diferentes estudios dieron cuenta de que América Latina ha atravesado durante las últimas décadas del siglo XX y lo que va del siglo XXI numerosas crisis que llevaron a interrupciones de los mandatos constitucionales presidenciales a través de situaciones con distinto grado de irregularidad.

De acuerdo a los estudios de María Eva Pignatta (2011) y de Carlos Closa Montero y Stefano Palestini Céspedes (2016), desde el año 1991 al año 2015, se sucedieron casi treinta crisis democráticas en América Latina, presentándose de distintas formas. Con algún grado de participación de las Fuerzas Armadas a través de Golpes de Estado aunque no seguidos por procesos dictatoriales en seis oportunidades (Haití 1991, 1994, 2004; Ecuador 2000; Venezuela 2002; Honduras 2009), por levantamientos militares en cuatro oportunidades (Venezuela 1992, Paraguay 1996, 2000; Ecuador 2010), a través de formas más novedosas como los autogolpes, en dos oportunidades (Perú 1992 y Guatemala 1993), las destituciones a través de juicios políticos en seis ocasiones (Brasil 1992, Venezuela 1993; Ecuador 1997, 2000, 2005; Paraguay 2012), las renuncias en cinco oportunidades (Paraguay 1999, Bolivia 2003, 2005; Haití 2004; Argentina 2001) los cuestionamientos electorales en tres oportunidades (República Dominicana 1994, Perú 2000, Haití 2001) y los casos de desestabilización y violencia política en dos oportunidades (Bolivia 2008, Venezuela 2015).

\section{La respuesta regional: los mecanismos de protección democrática en el marco de las organizaciones.}

A partir de la década de los años 90 del siglo pasado, y como modo de, por un lado, contribuir a la consolidación y protección de las democracias, y por otro lado, de prevenir posibles nuevas crisis democráticas, los Estados de América Latina se dieron en el marco de las organizaciones regionales ciertos instrumentos normativos con distinto grado de formalidad jurídica denominados "Mecanismos de protección democrática” (Pignatta, 2011; Canepa, 2015; Closa et al., 2016).

De acuerdo a la definición de Closa y otros (2016), estos mecanismos son las "reglas y procedimientos formales, semi-formalizados o informales a través de los cuáles las organizaciones regionales pueden intervenir en caso de una posible crisis democrática" (Closa et al., 2016: 20). Los mismos fueron adoptándose en forma de declaraciones, tratados, resoluciones y protocolos adicionales a los tratados constitutivos de las organizaciones regionales, y se constituyen como un cuerpo de posibles acciones a seguir por parte de los Estados miembros, en el ámbito del funcionamiento de los órganos de las mismas, en pos de prevenir, revertir o sancionar situaciones de amenaza, quebrantamiento e interrupción del normal funcionamiento del sistema democrático (Díaz, 2007; Trucco y Priotti, 2012; Canepa, 2015).

Si bien los mecanismos de protección democrática surgen luego de la transición a la democracia, fueron creados en dos etapas distintas. Una primera etapa la comprenden los años posteriores a la transición durante la década de los años noventa y primer año del siglo XXI (Pignatta, 2011; Closa et al., 2016). La misma fue testigo de la finalización de la guerra fría a nivel internacional y de la expectativa generada de que el sistema democrático se extendería indefectiblemente en todo el mundo acompañando el triunfo del capitalismo como modelo económico universal. (Frank, 1992; Perina, 2001; Pevehouse, 2005). 
Un segundo momento de creación de los mecanismos citados converge con un nuevo contexto regional caracterizado por la creación de nuevas organizaciones e instancias de coordinación política y con el reforzamiento de las ya existentes, durante la primera década de este siglo. Por ejemplo, el relanzamiento del MERCOSUR, y la creación de la UNASUR. Estos cambios respondieron a una nueva estrategia de integración regional más autónoma con respecto a los vínculos tradicionales de la región con Europa y EEUU (Bizzorero, 2011; Sanahuja, 2012; Kersffeld, 2013).

Una característica común a ambos momentos de creación de los mecanismos es que por un lado, fueron desarrollados y profundizados en escenarios de amenazas de crisis democráticas o de episodios concretos de quebrantamiento del orden democrático en algunos países de la región. Y por otro lado, fueron impulsados en el marco de una relación asimétrica con respecto a países en riesgo de crisis democrática y países que no lo estaban. El caso de Paraguay en el marco del MERCOSUR es esclarecedor con respecto a la adopción del Protocolo de Ushuaia y el Protocolo de Montevideo (Closa y Palestini, 2016).

En pos de describir los mecanismos es fundamental aclarar también que estas posibles acciones a seguir, sea para revertir situaciones de inestabilidad democráticas o para condenar las interrupciones del funcionamiento pleno del sistema político democrático y hasta para establecer algún tipo de sanciones, se desarrollan en ámbitos multilaterales cuyo basamento se asienta en órdenes jurídicos intergubernamentales que solo brindan herramientas facultativas. Es decir que para que esas herramientas sean puestas en acción, se hace necesario que se tomen decisiones por parte de los Estados miembros y que se logren consensos y/o mayorías, dependiendo del sistema de toma de decisiones de cada organización. Por lo tanto, el hecho de que las decisiones que impliquen la activación, verificación y sanción en el marco de los mecanismos deban someterse a tratamiento por parte de los Estados miembros, genera un amplio margen de discrecionalidad al momento de identificar los casos de crisis democrática, y sobre la necesidad de activar o no los mecanismos y de cómo hacerlo, si amerita o no aplicar sanciones, y sobre cómo verificar los procesos. Se genera así un marco de gran incertidumbre en la interpretación y aplicación de las normativas que contienen los mecanismos, y como consecuencia, el abordaje de las crisis democráticas es diferente en cada caso y puede responder a distintos factores. (Closa et al., 2016)

Este diseño institucional inspirado en un esquema intergubernamental con baja delegación de atributos en los órganos que activarán los mecanismos y con relativa precisión en conceptos trascendentales como qué se entenderá por el quiebre del sistema democrático o en qué casos concretos se estará en presencia de una crisis democrática, contiene un conjunto de atributos facultativos que obligarán a los Estados miembros en la medida de que estos acuerden y acepten las decisiones, generando un sistema precario y ambivalente de defensa de la democracia, características que se repiten en los instrumentos de todas las organizaciones regionales de la región, a saber: la OEA, el MERCOSUR, la UNASUR, la Comunidad Andina de Naciones (CAN), el Sistema de Integración Centroamericano (SICA) y la Comunidad del Caribe (CARICOM) (Closa et al, 2016).

\section{Dos destituciones, similitudes y diferencias de ambas crisis.}

La comparación de las crisis democráticas sucedidas en Paraguay (2012) y Brasil (2016) encuentra similitudes en principio al ser ambas crisis desencadenantes de dos juicios políticos seguidos de destituciones de sus mandatarios, y a su vez, al compartir ambos casos las organizaciones regionales competentes para actuar, a saber: la OEA, el MERCOSUR y la UNASUR.

Con respecto a los factores internos que producen las crisis vale rescatar las variables apuntadas por Aníbal Pérez Liñán (2009) en su estudio sobre los juicios políticos en América Latina. Para el autor "en un contexto de baja intervención militar, fueron las amplias protestas populares las que determinaron en gran medida, la caída de un gobierno. Pero fue sólo la presencia de los escándalos mediáticos y una legislatura proactiva lo que dio lugar al modelo institucional de remoción (...): el juicio político al presidente" (Pérez Liñán, 2009: 303)

Éstas características, a saber: baja participación militar, protestas multiclasistas demostrando descontento popular, escándalos mediáticos involucrando a las clases dirigentes y la conformación de una mayoría parlamentaria dispuesta a llevar adelante un proceso de remoción del presidente, están presentes en ambos casos aunque con algunos matices y particularidades.

Con respecto a la primera variable, la participación militar, si bien las fuerzas armadas habían tenido participación activa en toda la vida política paraguaya, con la larga dictadura de Stroessner como emblema, y también posteriormente a la transición democrática, con distintas crisis sucedidas con el intento de golpe de Estado contra el ex Presidente Wasmosy en el año 1996, el magnicidio contra el ex vicepresidente Luis María Argaña en el año 1999, y el fracasado levantamiento militar del año 2000 contra el 
Presidente Luis González Machi, en la crisis del año 2012 no hubo participación de las mismas. Lo mismo sucederá para el caso de Brasil (2016), ya que no aparece el factor militar en la crisis de la presidencia de Rousseff, ni es un actor al que se puede involucrar en la caída de la Presidenta, aún cuando las fuerzas armadas había dominado la vida política brasileña durante varias décadas previas a la transición democrática, y aún cuando esta transición se había desarrollado de forma pactada con este poder.

Con respecto al descontento popular, en el caso de $\mathrm{Pa}$ raguay, el mismo fue generado en parte por la acción de los medios de comunicación involucrando en escándalos personales al presidente, aunque también se alimentaba con la cuestión de la inseguridad que afectaba a las clases apoderadas que temían por la ocupación de tierras. La masacre de Curuguaty, que involucró la represión de grupos campesinos ocupando un predio de tierras fiscales, fue la excusa para apuntar a un presidente debilitado en su alianza de gobierno alimentándose la idea del gobierno débil e ineficiente. A su vez, las decisiones de política exterior del mandatario que involucraron la aceptación del ingreso de Venezuela al MERCOSUR, a sabiendas de la oposición tanto del Partido Colorado como del Liberal en su representación en el Congreso, y la firma del Tratado de Montevideo en el marco de ese bloque, sumaron para el descontento tango en su espacio de gobierno como en la oposición (Soler; 2013; Yussef, 2013).

La alianza de gobierno de Lugo con el Partido Liberal que había llegado al poder rompiendo la hegemonía de más de 60 años del Partido Colorado termina quebrándose y los liberales pasan a quitarle el apoyo. Sin una fuerza política propia y habiendo construido poco al respecto Lugo es sometido a una nueva mayoría parlamentaria tanto en la Cámara de Diputados como en la de Senadores que se dispuso a acusarlo y a enjuiciarlo de forma express y con la aprobación casi absoluta en ambos recintos.

Por su parte en Brasil, el descontento popular provenía más de la crisis económica que el país estaba atravesando, la Presidenta tomó medidas de ajuste que había planteado su contrincante en las elecciones y esto repercutió hasta en su propio electorado. Rousseff, a diferencia de Lugo, representaba un proyecto en el poder que encabezó Lula da Silva a partir del año 2002 y que ella continuó, pero que comenzaba a atravesar un momento de evidente desgaste. Esto se vio reflejado en el resultado de las elecciones que le habilitan el segundo mandato con una victoria con margen bastante acotado frente a Aecio Neves. Además, las denuncias por corrupción que apuntaban a casi la totalidad de la clase política, aunque con la excepción de la
Presidenta, sumaban y ampliaban el descontento popular por la mala performance de la economía, produciéndose grandes manifestaciones multiclasistas por todas las grandes urbes.

También en el caso de Brasil estamos ante un quiebre del espacio gobernante integrado por el Partido de los Trabajadores y los otros partidos integrantes que le comienzan a quitar el apoyo, en especial el Partido del Movimiento Democrático Brasilero del que forma parte el Vicepresidente, Michel Temer. La baja popularidad resaltada por los grandes medios de comunicación se suma al descontento de la gente por la corrupción y la crisis económica, generando un escenario de pérdida de legitimidad presidencial que le da impulso a la conformación de una nueva mayoría en el Poder Legislativo decidida a destituir a la Presidenta como un camino para superar la fuerte crisis política en la que se sumergía el país.

Ambos procesos de acusación y juzgamiento de los mandatarios tuvieron en común que se le detectaran irregularidades que fueron reconocidas por actores internos del sistema y por actores externos, con respecto a estos últimos tanto por los Estados de la región de forma unilateral, como por parte de las organizaciones regionales. Ahora bien, se hace necesario distinguir y caracterizar estos procesos irregulares porque también cuentan con matices propios.

Por un lado, la destitución de Fernando Lugo fue un proceso extremadamente corto, en menos de 24 horas se acusa y se juzga al Presidente, no respetando el debido proceso, es decir, el tiempo necesario para una debida defensa en juicio. A su vez, la acusación que es expresada en menos de una carilla, se basaba en las responsabilidades por la masacre de Curuguaty y por las decisiones de política exterior del presidente que se consideraban que habían puesto en riesgo al país (Soler, 2013; Kersffeld, 2013).

El proceso de destitución de Dilma Rousseff, si bien no fue un proceso express, ya que se respetaron los tiempos necesarios para la defensa, incluyó irregularidades que estuvieron relacionadas con las causas que escoge el Parlamento como fundamento para destituir a la mandataria. En este sentido, la acusación se elabora en base a acciones administrativas relacionadas a modificaciones en las partidas presupuestarias, medidas que son tomadas habitualmente por los mandatarios en el poder y que no tendrían que constituir causal para un juicio político, pero en este caso lo fueron. 
De esta forma, lo que sí sucedió en ambos juicios como característica fundamental fue la conformación de nuevas mayorías parlamentarias, y por consiguiente, la pérdida del escudo legislativo de ambos presidentes, que más allá de las irregularidades en el desarrollo de los procesos y de las causas establecidas para acusarlos, tenían como objetivo y estaban decididas a sacar a los mandatarios del poder. Episodios que pueden ser considerados ejemplos en un nuevo patrón de inestabilidad en la región, que en palabras de Tokatlian se denominan "neogolpismos", "golpes benévolos" o "golpes camuflados", que involucran a los mismos poderes del Estado, como la actitud proactiva del Poder Legislativo contra el Poder Ejecutivo y la complicidad del Poder Judicial que corrobora la legalidad de la situación y simula el respeto por la institucionalidad. Según el autor, los mismos son parte de un proceso gradual que tiene por objeto superar impasses políticos justificando la acción antidemocrática que pueden llegar a producir en la necesidad de salvar situaciones de autoritarismo, vacío de poder, crisis autoproducidas e intención desmedida de poder y de perpetuación en el mismo. ${ }^{1}$

En línea con estas apreciaciones, Lorena Soler adhiere a la existencia de nuevas formas de Golpes de Estado en América Latina relacionadas con acciones restauradoras del statu quo anterior a las reformas que fueron llevando adelante gobiernos como el de Lugo, y agrega que la ambigüedad que presentan permite disfrazarlos de continuidad institucional y disimularlos (Soler, 2015).

\section{La asimetría de poder y los contextos regionales como factores determinantes en la acción de las organizaciones}

En el caso de Paraguay (2012) y Brasil (2016), se observan dos casos de crisis democráticas que a pesar de sus propias particularidades presentan características similares, fundamentalmente en la forma en que se interrumpe el normal funcionamiento de las instituciones democráticas, a saber: el juicio político y la destitución de los presidentes de forma irregular. Ahora bien, ¿cuáles fueron los factores que determinaron el accionar marcadamente disímil de las organizaciones regionales ante las mismas?
Tal como se plantea con anterioridad, la estructura jurídica intergubernamental de las organizaciones regionales, y la consecuente necesidad de discutir cada caso práctico para la toma de decisiones correspondientes en la aplicación de los mecanismos de protección democrática de las organizaciones regionales, impacta en el accionar de las mismas dejando márgenes para una interpretación ambigua y un comportamiento discrecional por parte de los Estados miembros, abriendo la puerta a la influencia de factores que inciden en la acción de estos entes con respecto a las distintas crisis. Los dos casos que se comparan en este artículo dan cuenta de esta conjetura.

Siguiendo a las autoras Andrea Ribeiro Hoffmann y Anna van Der Vleuten (2010) el principal factor de impacto en la intervención de una Organización Regional en un caso de crisis democrática, se encuentra en el rol que juegan los poderes globales y regionales con respecto al mismo. Es decir, la postura que los países líderes tengan frente a la crisis y la presión que ejerzan para que se tome una decisión determinada en los ámbitos multilaterales es clave para hacer converger una postura grupal en el marco de las organizaciones. Los Estados que son considerados líderes en una región sobresalen en distintas dimensiones que se relacionan con la capacidad de preservación y fortalecimiento de su poder, de transferencia de sus valores y de conservación de sus riquezas, y a su vez, son reconocidos por los demás países como tales. El estudio de las autoras involucra casos de crisis democráticas de América Latina, donde se reconoce el rol de líderes a EEUU en el marco de la OEA, y a Brasil en el marco del MERCOSUR, estudio de casos realizado previamente a la creación de UNASUR (Ribeiro Hoffman y Van der Vleuten, 2010).

La asimetría en la percepción de inestabilidad entre los países miembros de las organizaciones regionales, y como consecuencia la probabilidad de aplicación o no de los mecanismos de protección democrática dependiendo de esta condición es otro factor trascendental, que junto al impacto de los contextos políticos regionales, definido por la afinidad entre los gobiernos, inciden en el accionar frente a las crisis democráticas, y en la interpretación y aplicación de los mecanismos de protección democrática (Ribeiro Hoffman y Van der Vleuten, 2010; Ribeiro Hoffmann, 2015; Closa y Palestini, 2016; Palestini, 2017). 
Por un lado, en el caso de Paraguay hubo una clara convergencia entre la acción del MERCOSUR y la UNASUR que decidieron que la destitución del Presidente Lugo fue un quiebre del sistema democrático paraguayo, al considerar que el juicio político que se le realizó fue sin respeto alguno por la debida defensa y estuvo impulsado por causas poco fundamentadas (Gallardo, 2012; Soler, 2013; Yussef, 2013).

Ambas organizaciones consideraron aplicar las sanciones contempladas en sus Protocolos sobre protección democrática. En el caso de la UNASUR, se inicia una misión verificadora de los hechos, y se decide la suspensión de Paraguay, quitándole el derecho de participación en el ámbito de sus órganos. A su vez, se establece una Unidad Especial para el seguimiento del futuro proceso eleccionario. La aplicación de la suspensión genera suspicacias al momento de saberse la falta de entrada en vigor del Protocolo Adicional al Tratado Constitutivo de UNASUR sobre Compromiso con la Democracia de noviembre de 2010, aunque la decisión puede fundamentarse en el rol político por el cual se había creado la organización: la contención y resolución de las crisis políticas para preservar la democracia, objetivo plasmado en el Tratado Constitutivo de la organización. ${ }^{2}$

El MERCOSUR que por voluntad política de los miembros coordinaba sus acciones con la UNASUR, ya que las Cumbres de Jefes y Jefas de Estado de ambas organizaciones se hacían en esos años en las mismas fechas aprovechando la coincidencia de los miembros plenos y asociados del bloque con las partes de la organización sudamericana, decide suspender a Paraguay de la participación en sus órganos, aunque no así de los proyectos de convergencia económica de los que formaba parte, como forma de sancionar a los poderes del Estado y no a la población. De todas formas, la decisión de suspensión fue publicada por la Cancillería de Argentina, que contaba con la presidencia Pro Tempore del bloque, cinco días previos a la realización de la Cumbre en Mendoza el 29 de junio de 2012, no coincidiendo con lo estipulado sobre la forma de adoptar la decisión de acuerdo al Protocolo de
Ushuaia que establece el deber de realizarse en el marco de una sesión ampliada del Concejo de Mercado Común y posteriormente a la realización de consultas. ${ }^{3}$

La OEA, por su parte, toma conocimiento de la realización del juicio político y de la forma extremadamente rápida en que se realiza. El Concejo Permanente se reúne y decide enviar una misión verificadora al mando del Secretario General José Miguel Inzulza, acompañado por representantes de países miembros, ninguno sudamericano. Una vez en el lugar la misión se entrevista con todos actores internos involucrados y llega a la principal conclusión de que el proceso no se había realizado con todas las garantías. El informe de la misión es presentado en el Concejo Permanente y los representantes de los países debaten sobre la situación, se observan diferentes posturas desde las que claramente reconocían el quiebre del sistema democrático y otras que minimizaban la situación. Finalmente no termina primando ninguna decisión mayoritaria como para activar otro tipo de mecanismos como podría haber sido la Reunión de Ministros de Relaciones Exteriores, y la decisión de suspensión de Paraguay de la organización de acuerdo a lo estipulado en la Carta Democrática y actuando en coordinación con las otras organizaciones. ${ }^{4}$

En este sentido llama la atención el rol de EEUU, por un lado, activo en la participación en la misión verificadora y en el reconocimiento de la irregularidad de la situación, pero por otro lado, pasivo en su capacidad de impulsar decisiones al respecto. Esto difiere claramente con su accionar en las crisis de Paraguay durante la década de los años 90, en las cuáles tanto el Embajador de ese entonces en Asunción, como el representante del Departamento de Estado jugaron fuertemente a favor de revertir las crisis de la Presidencia de Wasmosy y de Cubas Grau. Brasil y Argentina, en su rol de potencias regionales habían jugado todas sus cartas a través de las iniciativas de sus mandatarias en el ámbito del MERCOSUR y la UNASUR, y si bien sus posturas fueron de condena en el marco de la OEA junto a otros países como Venezuela, Nicaragua, Perú y Bolivia, no se mostraron interesadas tampoco en promo-

2. Ver Preámbulo y artículo 2 del Tratado Constitutivo de la Unión de Naciones Sudamericanas, suscrito en Brasilia, República Federativa de Brasil, 23 de mayo de 2008. Disponible en: http://www.unasursg.org/images/descargas/DOCUMENTOS\%20CONSTITUTIVOS\%20DE\%20UNASUR/Tratado-UNASUR-solo.pdf. Consulta: 15 de mayo de 2018..

3. Ver artículos 4, 5 y 6 del Protocolo de Ushuaia, suscrito en Ushuaia, República Argentina, el 24 de julio de 1998. Disponible en http://www.mercosur. int/t_generic.jsp? contentid $=2485 \&$ site $=1 \&$ channel $=$ secretaria. Consulta: 27 de mayo de 2018 .

4. Informe de la misión del Secretario General de la OEA y la Delegación a la República del Paraguay, 10 de julio de 2012, Washington. Disponible en: DChttp://www.oas.org/es/acerca/discurso_secretario_general.asp?sCodigo=12-0057. Consulta: 23 de mayo de 2018. 
ver una decisión mayoritaria en el marco de este espacio. Tampoco los integrantes de la misión que envía la organización, Canadá y EEUU entre otros, utilizaron lo recabado por ellos mismos para promover algún tipo de acción, ni se mostraron proclives a generar una decisión en sintonía con las otras dos organizaciones involucradas.

En el caso de Brasil las organizaciones actuaron muy diferente al caso Paraguayo. En primer lugar, el MERCOSUR ni siquiera se pronuncio al respecto, ni al momento de la acusación de la Presidenta por parte de la Cámara de Diputados que implicó la suspensión en el ejerció de su cargo, ni al momento del juzgamiento y la consecuente destitución. Sí hubo declaraciones unilaterales de las partes al respecto, y se resalta en este caso la postura de Argentina que se expresó reconociendo el nuevo Gobierno de Temer inmediatamente y sin ningún cuestionamiento del proceso.

La UNASUR por su parte, venía atravesando una etapa de inactividad significativa, la reunión ordinaria del Concejo de Jefes y Jefas de Estado, órgano máximo que se reúne anualmente, no se reunía desde fines del año 2014. Recién en abril de 2016, en la reunión del Concejo de Ministros de Relaciones Exteriores se trató la cuestión de que la Presidenta había sido suspendida en sus funciones y restaba el proceso de juicio por parte del Senado. La delegación brasileña trasladó la preocupación y la voluntad de consensuar una decisión, sin embargo, la Canciller Argentina Susana Malcorra y su par de Colombia María Ángela Olguín relativizaron la situación favoreciendo que el plenario no se resuelva por tomar una postura al respecto. La única acción concreta de la Organización se vio plasmada, por un lado, en las declaraciones del Secretario General Ernesto Samper, que más allá de no contar con atributos para activar los mecanismos siguió de cerca el proceso constatando las irregularidades del mismo publicando extensos comunicados de prensa al respecto, y por otro lado, las declaraciones del Presidente Pro Tempore Nicolás Maduro que en el momento de la destitución fueron a favor de reconocer que se estaba tratando de un quiebre institucional pero también reconociendo que no se habían dado los consensos necesarios entre los miembros para realizar la reunión de Jefes y Jefes de Estado y generar algún tipo de iniciativa.
Desde la OEA, la labor del Secretario General Luis Almagro se activó al recibir la notificación del Gobierno de Brasil solicitando que se realice un seguimiento del proceso y se activen los mecanismos de protección tal como lo estipula la Carta Democrática en sus artículos 17 y 18. De esta forma, el funcionario encabezó dos misiones verificadoras tanto al momento de la suspensión de la Presidenta como en los días previos y durante la destitución. En las misiones pudo corroborar la irregularidad de los hechos al haberse destituido a un mandatario por acciones administrativas propias de sus labores, procediendo a publicar sendos comunicados de prensa al respecto. Sin embargo, llamativamente no se registra en las actas del Concejo Permanente que los informes de las misiones se hayan por lo menos presentado en el marco del mismo. ${ }^{6}$

\section{Conclusión}

Este artículo dio cuenta de la inestabilidad política y de las recurrentes crisis democráticas que se produjeron en la región con golpes de Estado y dictaduras durante todo el siglo veinte, y de nuevas formas de inestabilidad que se vienen produciendo a partir de la transición democrática en la década de los años 80. Estas crisis democráticas post transición llevaron a los Estados, en el marco de las organizaciones regionales, a la creación de mecanismos de protección democrática en pos de actuar frente a estas situaciones ejerciendo iniciativas para disuadirlas o revertirlas, y para como una vía para consolidar las democracias latinoamericanas. Con mayor o menor grado de formalidad estos mecanismos poco precisos y de bajo grado de delegación, se asentaron a su vez en órdenes jurídicos intergubernamentales los cuáles brindan amplios márgenes de acción a los Estados generando discrecionalidad a la hora de aplicarlos en los casos de crisis democráticas.

Se observaron específicamente dos casos concretos de crisis democráticas que acarrearon las destituciones de dos mandatarios de forma irregular, Fernando Lugo en Paraguay en al año 2012, y Dilma Rousseff en Brasil en el año 2016, y se analizó cuál fue la respuesta regional a ambas crisis considerando la acción de las organizaciones regionales competentes, a saber: la OEA, el MERCOSUR y la UNASUR.

5. Carta Democrática Interamericana de la OEA. Disponible en: http://www.oas.org/OASpage/esp/Documentos/Carta_Democratica.htm. Consulta: 25 de junio de 2018.

6. Actas de las sesiones del Consejo Permanente de la OEA. Disponible: http://www.oas.org/es/council/CP/documentation/minutes. Consulta: 15 de mayo de 2018 
En el caso de Paraguay (2012) se observó un contexto regional de convergencia en la acción y de afinidad en la consideración de todos los mandatarios de la región sobre la necesidad de actuar en el marco de situaciones de quiebre democrático, destacándose las iniciativas de Brasil en el marco de la UNASUR y de Argentina en el marco del MERCOSUR con la aplicación de la suspensión de Paraguay de la participación en ambas organizaciones hasta que se realicen elecciones libres y competitivas y se restablezca el orden democrático. El rol de la OEA, en cambio, no fue el de acompañar y coordinar con estas organizaciones a pesar de que la misión verificadora, ordenada en al marco del Concejo Permanente, llegara a las mismas conclusiones considerando al proceso como notoriamente arbitrario por el escaso tiempo en el que se desarrolló, sin las debidas garantías para la defensa del mandatario y con causales de relativo peso, aunque excusándose en que el Poder Judicial no había declarado la inconstitucionalidad del mismo. La falta de iniciativa de EEUU en el marco de dicha organización, se enmarca en un contexto regional adverso promovido por los gobiernos no afines y desafiantes a sus políticas en la región, que actuaban con un margen de autonomía en los espacios multilaterales como la UNASUR.

En el caso de Brasil (2016), la percepción asimétrica entre los Estados partes de las organizaciones regionales donde algunos Estados son considerados candidatos a aplicárseles los mecanismos por su condición de vulnerables, mientras otros ostentan el rol de líderes que tienen iniciativa política para la aplicación de los mecanismos, jugó a favor de la improbabilidad de que estos se aplicarán al gigante regional. El rol de Argentina como potencia regional secundaria fue clave en aceptar el proceso de destitución, y apoyarlo al reconocer de inmediato al Gobierno de Temer. Esto se vio reflejado en la falta de tratamiento del tema en la órbita del MERCOSUR y en la falta de decisiones de la UNASUR a pesar de la solicitud del gobierno brasileño de activar los mecanismos. El rol de EEUU en el marco de la OEA, aunque en un contexto regional menos desfavorable auspiciado con el cambio de gobierno en Argentina en diciembre de 2015, fue el de dilatar la situación y no considerar necesario impulsar una iniciativa con respecto a la situación de Brasil, a pesar de los informes recabados durante las misiones realizadas por el Secretario General Luis Almagro que constataron la arbitrariedad e irregularidad del proceso con respecto a las causales de acusación y enjuiciamiento.

Como conclusión y sin negar el impacto positivo de la creación de mecanismos de protección democrática como herramientas de las organizaciones para prevenir y san- cionar posibles crisis, la estructura institucional en la que se asientan genera un margen de incertidumbre sobre los casos de crisis democrática que se identifican, y las decisiones que se toman al respecto, favoreciendo así la influencia de factores como la asimetría de poder entre los Estados miembros y los diversos contextos políticos regionales a la hora de proteger o no el sistema democrático de gobierno, como ha quedado demostrado en el ejercicio comparativo de los dos casos de crisis retomadas aquí.

\section{Bibliografía}

Actas de las sesiones del Consejo Permanente de la OEA. Disponible: http:// www.oas.org/es/council/CP/documentation/minutes/. Consulta: 15 de mayo de 2018

Acta de la Sesión Extraordinaria del Consejo Permanente de la OEA, viernes 22 de junio de 2012. Consultado el 10 de mayo de 2018. Disponible en: http://www.oas.org/es/council/CP/documentation/minutes/. Consulta: 10 de mayo de 2017.

Acta de la Sesión Extraordinaria del Consejo Permanente de la OEA. Celebrada el 15 de Abril de 2016. Disponible: http://www.oas.org/es/council/CP/documentation/minutes/. Consulta: 20 de mayo de 2017.

BIZZORERO, L. (2011); "América Latina a inicios de la segunda década del siglo XXI: entre el regionalismo estratégico y la regionalización fragmentada", Revista Brasilera de Política Internacional, vol.54, nro.1, Brasilia. Disponible en: http://www.fes-seguridadregional.org/index. php?option=com_booklibrary\&task $=$ view $\&$ id $=4018 \&$ catid $=262 \&$ Ite mid=319. Consulta: 10 de abril de 2018 .

CANEPA, M. (2015); "Las cláusulas democráticas en los procesos de integración latinoamericanos", Revista Iberoamericana de Derecho Internacional y de la Integración. Disponible en: http://www.pensamientocivil.com.ar/system/files/2015/08/Doctrina1674.pdf\#viewer. action=download. Consulta: 8 de julio de 2018 .

Carta Democrática Interamericana de la OEA. Disponible en: http://www. oas.org/OASpage/esp/Documentos/Carta_Democratica.htm. Consulta: 25 de junio de 2018

CLOSA, C., CASTILLO, P., y PALESTINI, S.; (2016); “Organizaciones Regionales y Mecanismos de Protección de la Democracia en América Latina, el Caribe y la Unión Europea"; Fundación EU-LAC, Hamburgo, Alemania.

CLOSA C. y PALESTINI S., (2016); "Defensa de la democracia o au- 
todefensa: las organizaciones regionales y la protección de la democracia en América Latina y el Caribe", Pensamiento Propio $\mathrm{N}^{\circ}$ 44, pp. 145-146. Disponible en: http://www.cries.org/wp-content/ uploads/2017/02/010-closa.pdf. Consulta: 20 de junio de 2018.

Comunicado de Prensa, Consejo Permanente de la OEA recibió informe del Secretario General y delegación a Paraguay, 10 de julio de 2012. Disponible en: http://www.oas.org/es/centro_noticias/comunicado_ prensa.asp?sCodigo=C-247/12. Consulta: 20 de junio de 2018 .

Comunicados de Prensa de la Secretaría General de la OEA del mes de mayo de 2016. Disponible en: http://www.oas.org/es/centro_noticias/ comunicados_prensa.asp?nMes $=5 \&$ nAnio $=2016$. Consulta: 26 de mayo de 2018.

Comunicado de Prensa de la Secretaría General de la OEA, Situación de Brasil, 15 de abril de 2016. Disponible en: http://www.oas.org/es/centro_noticias/comunicado_prensa.asp?sCodigo=C-044/16. Consulta: 27 de mayo de 2018.

Comunicado de Prensa, Secretario General de la OEA llama a que se respete el debido proceso en Paraguay, 21 de junio de 2012. Disponible en: http://www.oas.org/es/centro_noticias/comunicado_prensa.asp?sCodigo=C-226/12. Consulta: 23 de junio de 2018.

Comunicado de Prensa, Secretario General de la OEA llama a resguardar el diálogo y el entendimiento en Paraguay, 21 de junio de 2012. Disponible en: http://www.oas.org/es/centro_noticias/comunicado_prensa. asp?sCodigo=C-223/12. Consulta: 23 de mayo de 2018.

Comunicado de Prensa, Secretario General de la OEA plantea dudas sobre respeto de legítima defensa en juicio político que destituyó a ex Presidente de Paraguay, 23 de junio de 2012. Disponible en: http://www.oas. org/es/centro_noticias/comunicado_prensa.asp?sCodigo=C-229/12. Consulta: 23 de junio de 2018.

Comunicado de Prensa, Secretario General de la OEA viajará a Paraguay para recabar información sobre recientes acontecimientos, 26 de junio de 2012. Disponible en: http://www.oas.org/es/centro_noticias/comunicado_prensa.asp?sCodigo=C-234/12. Consulta: 23 de mayo de 2018.

Comunicado de la Secretaria General de UNASUR, 18 de abril de 2016. Disponible en: http://www.resumenlatinoamericano.org/wp-ontent/ uploads/2016/04/12983986_1099422156766557_52923462828522612 47_o.jpg. Consulta: 24 de mayo de 2018.

Comunicado de Prensa de la Cancillería Argentina sobre el Proceso Institucional en Brasil, 31 de agosto 2016. Disponible en: https://www.mrecic. gov.ar/proceso-institucional-en-brasil. Consulta: 26 de mayo de 2018.

Comunicado de Prensa de la UNASUR sobre la suspensión de Dilma Rousseff, 12 de mayo de 2017. Disponible en: https://www.youtube.com/ watch?v=UxMWpQDJbsk. Consulta: 18 de mayo de 2018
Decisión de la Reunión Extraordinaria del Concejo de Jefes y Jefas de Estado y de Gobierno de UNASUR. Mendoza, República Argentina, 29 de junio de 2012. Disponible en: https://repo.unasursg.org/alfresco/ service/unasursg/documents/content/DECISION_No_26_2012_ REUNION_EXTRAORDINARIA_DEL_CONSEJO_DE_JEFAS_Y_ JEFES_DE_ESTADO_Y_DE_GOBIERNO_DE_UNASUR.pdf?noderef $=53 \mathrm{~d} 2 \mathrm{a} 27 \mathrm{e}-1 \mathrm{bf5}-434 \mathrm{c}-8 \mathrm{c} 94-0 \mathrm{~b} 1 \mathrm{e} 721 \mathrm{a} 832 \mathrm{e}$. Consulta: 25 de mayo de 2018.

Decisión sobre la suspensión del Paraguay en el MERCOSUR, Mendoza, 29 de junio de 2012.. Disponible en: https://www.mrecic.gov.ar/ print/246081. Consulta: 3 de junio de 2018]

Declaración de la Reunión Ordinaria del Concejo de Ministras y Ministros de Relaciones Exteriores de UNASUR, Quito, 23 de abril de 2016. Disponible en: https://repo.unasursg.org/alfresco/service/unasursg/ documents/content/DECLARACION_DE_LA_REUNION_ORDINARIA_DEL_CONSEJO_DE_MINISTRAS_Y_MINISTROS_ DE_RELACIONES_EXTERIORES_DE_UNASUR_QUITO_23_ ABRIL_2016.pdf?noderef=7a5a894d-9b3a-433f-a233-a0aaf8e2f7d2. Consulta: 24 de mayo de 2018.

Declaraciones y Decisiones Presidenciales del Mercosur. Disponible en: http://www.mercosur.int/innovaportal/v/4506/11/innova.front/declaraciones-y-decisiones-presidenciales. Consulta: 10 de junio de 2018.

DIAZ, C. (2007); "Las clausulas democráticas en los Tratados internacionales. La experiencia en la crisis de la democracia paraguaya en 1996"; Tesis de Maestría FLACSO, Buenos Aires.

GALLARDO, R., (2012); "Paraguay: el detalle de un golpe de estado Express” En Grupo de Estudios Internacionales Contemporáneos, Córdoba. Disponible en: http://www.geic.com.ar/2010/2012/06/24/ paraguay-causas-y-consecuencias-de-ungolpe-de-estado-express/. Consulta: 1 de junio de 2018.

Informe de la misión del Secretario General de la OEA y la Delegación a la República del Paraguay, 10 de julio de 2012 - Washington. Disponible en: DChttp://www.oas.org/es/acerca/discurso_secretario_general.asp?sCodigo=12-0057. Consulta: 23 de mayo de 2018.

KERSFFELD, D. (2013); El papel de la Unasur ante los conflictos internacionales: dos estudios de caso. Revista Mexicana de Ciencias Políticas y Sociales, 58 (218), 193-208. Disponible en: http://ac.els-cdn.com/ S0185191813722967/1-s2.0-S0185191813722967-main.pdf?_tid=dd530fcc-4e61-11e7-90cd-00000aab0f02\&acdnat=1497156969_dc084f87d411e0204f546c27dedfd510. Consulta: 2 de mayo de 2018.

MARSTEINTREDET, L., LLANOS, M. y NOLTE, D.; (2013) Paraguay and the Politics of Impeachment. Journal of Democracy 24(4): 110-123. Disponible en: https://www.giga-hamburg.de/sites/default/files/publications/article_nolte_llanos_marsteintredet.pdf. Consulta: 20 de abril de 2018. 


\section{La destitución de Fernando Lugo y Dilma Rousseff. \\ Una comparación de ambas crisis democráticas desde la labor de las organizaciones regionales.}

Ministerio de Relaciones Exteriores y Culto de la República Argentina. Información para la Prensa $\mathrm{N}^{\circ}: 210 / 12$, El MERCOSUR suspende a Paraguay, 24 Junio 2012. Disponible en: http://www.mrecic.gov.ar/es/ el-mercosur-suspende-paraguay. Consulta: 1 de junio de 2018.

PALESTINI, S. (2017); “Defender la democracia en América Latina... pero ¿qué democracia?” 15 Febrero. Disponible en: https://www.opendemocracy.net/democraciaabierta/stefano-palestini/defender-la-democracia-en-am-rica-latina-pero-qu-democracia. Consulta: 20 de mayo de 2018.

PEREZ LIÑAN, A. (2009); "Juicio político al presidente y nueva inestabilidad política en América Latina", Fondo de Cultura Económica, Buenos Aires. Capítulo VII.

PERINA, R. (2001); "El Régimen Democrático Interamericano: el papel de la OEA”. Disponible en: http://www.oas.org/sap/publications/2001/art/ art_002_01_spa.pdf. Consulta: 1 de mayo de 2018.

PEVEHOUSE, J. (2005); “Democracy from Above, Regional organizations and democratization”, Cambridge University Press, United Kingdom.

PIGNATTA, M. E. (2008); "La defensa de la democracia en la OEA. Nuevos instrumentos y debates"; Cuaderno de Cátedra; Política Internacional Latinoamericana; FCPOLIT-UNR; Rosario.

PIGNATTA, M. E. (2010); "Acerca de la defensa de la democracia en la OEA Condicionantes históricos y evolución reciente"; FCPOLIT-UNR-CONICET; Rosario. IX Congreso Nacional y II Congreso Internacional sobre Democracia.

PIGNATTA, M. E. (2011); "Multilateralismo y defensa de la democracia: el accionar de la Organización de Estados Americanos frente a crisis político-institucionales (1990-2009)", Capítulo 3. Tesis doctoral, FCPOLIT-UNR, Rosario.

PRIOTTI, A. y TRUCCO, M. (2012); "La clausula democrática en los procesos de integración latinoamericana”, Revista RecorDIP, Volumen 2, $\mathrm{N}^{\circ} 2$. Disponible en: http://www.cries.org/wp-content/ uploads/2017/02/010-closa.pdf. Consulta: 30 de octubre de 2017.

Protocolo Adicional al Tratado Constitutivo de UNASUR sobre compromiso con la Democracia, suscrito en Georgetown, República Cooperativa de Guyana, 26 de noviembre de 2010. Disponible en: http://www. unasursg.org/images/descargas/DOCUMENTOS\%20CONSTITUTIVOS\%20DE\%20UNASUR/Protocolo-Adicional-al-Tratado-Constitutivo-de-UNASUR-sobre-Compromiso-con-la-Democracia-opt.pdf. Consulta: 27 de mayo de 2018.

Protocolo de Ushuaia, suscrito en Ushuaia, República Argentina, el 24 de julio de 1998. Disponible en http://www.mercosur.int/t_generic.jsp?contentid=2485\&site=1\&channel=secretaria. Consulta: 27 de mayo de 2018.

RIBEIRO HOFFMANN, A. (2015); “As Organizaçöes Regionais e a Pro- moçäo e Proteçäo da Democracia: reflexões a partir das práticas de intervenção democrática na América do Sul", Pontificia Universidade Católica do Rio de Janeiro. Departamento de Relações Internacionais. Caderno CRH, Salvador, v. 29, n. SPE 03, p. 47-57. Disponible en: http:// www.scielo.br/pdf/ccrh/v29nspe3/0103-4979-ccrh-29-spe3-0047.pdf. Consulta: 15 de mayo de 2018.

SANAHUJA, J.A. (2012); "Post-liberal regionalism in South America: the case of UNASUR”. EUI Working Papers. RSCAS 2012/05. Disponible en: http://cadmus.eui.eu/bitstream/handle/1814/20394/RSCAS_2012_05.pdf?sequence=1\&isAllowed $=$ y. Consulta: 30 de mayo de 2018 .

SOLER, L. (2013); "Elecciones en Paraguay. Una transición política inacabada." Revista El Dipló, Edición Nro. 166.

SOLER, L. (2015); “Golpes de Estado en el siglo XXI. Un ejercicio comparado Haití (2004), Honduras (2009) y Paraguay (2012)”, Universidad de Buenos Aires, Buenos Aires, Argentina.

TOKATLIAN, Juan Gabriel, (2009) “Neogolpismo”, Diario Página 12, Buenos Aires, 13 de julio. Disponible en: https://www.pagina12.com.ar/ diario/elmundo/subnotas/128159-41146-2009-07-13.html. Consulta: 20 de abril de 2018.

TOKATLIAN, Juan Gabriel (2012) “El Auge del Neogolpismo”, Diario La Nación, Buenos Aires, 24 de junio. Disponible en: http://www.lanacion.com.ar/1484794-el-auge-del-neogolpismo. Consulta: 20 de abril de 2018.

Tratado Constitutivo de la Unión de Naciones Sudamericanas, República Federativa de Brasil, el 23 de mayo de 2008. Disponible en: http://www. unasursg.org/images/descargas/DOCUMENTOS\%20CONSTITUTIVOS\%20DE\%20UNASUR/Tratado-UNASUR-solo.pdf. Consulta: 15 de mayo de 2018.

VALENZUELA, A. (2004); "Latin American Presidencies Interrupted", Journal of Democracy, volumen $15, \mathrm{~N}^{\circ} 4$. Disponible en:

http://www.journalofdemocracy.org/sites/default/files/Valenzuela-15-4. pdf. Consulta: 1 de abril de 2017.

VLEUTEN, V. D. A. y HOFFMANN, A.R. (2010); Explaining the Enforcement of

Democracy by Regional Organizations: Comparing EU, Mercosur and SADC. Journal of Common Market Studies 48(3): 737-758. Disponible en: file://C:/Users/i-mag/Downloads/SSRN-id1600980.pdf . Consulta: 20 de mayo de 2018.

YUSSEF, N. (2013); "Neogolpismo: el caso Paraguayo", Centro de Estudios Políticos e Internacionales, CEPI, La Plata. Disponible en http://www. iri.edu.ar/images/Documentos/CENSUD/boletines/41/art_yuseff.pdf. Consulta: 15 de mayo de 2018.

Recibido 30/07/2018 - Aceptado 10/11/2018 